CASE REPORT

\author{
J.B. White \\ H.J. Cloft \\ D.F. Kallmes
}

\section{But Did You Use HydroCoil? Perianeurysmal Edema and Hydrocephalus with Bare Platinum Coils}

SUMMARY: Bioactive coils were developed to improve aneurysm packing and accelerate neointimal healing. Recent reports, however, have implicated these coils in the development of aseptic meningitis, perianeurysmal edema, and hydrocephalus. This report demonstrates that the same clinical phenomena can occur with the use of bare platinum coils.
$H^{i}$ igh recurrence rates following coil embolization with bare platinum led to the development of second-generation "bioactive" coils. Coincident with increased use of these newer coils has been concern over untoward effects on the surrounding CSF and brain parenchyma. Recent reports have implicated newer coils in the development of aseptic meningitis, perianeurysmal edema, and hydrocephalus in the postprocedural setting. ${ }^{1-3}$

This report presents 2 patients whose aneurysms were treated with bare platinum coils who subsequently developed significant brain stem edema and hydrocephalus.

\section{Case Reports}

\section{Case 1}

A 73-year-old man presented with an incidentally discovered right vertebral artery aneurysm that measured $17 \times 17 \times 15 \mathrm{~mm}($ Fig $1 \mathrm{~A})$ as detected on CT angiography. The pretreatment CT scan revealed no evidence for pre-existing cerebral or brain stem edema. He was premedicated with aspirin and clopidogrel. Under general anesthesia, the patient underwent coiling of the aneurysm with a total of 17 bare platinum coils from 3 different manufacturers (Orbit Trufill, Cordis, Miami Lakes, Fla; HeliPaq, Micrus Endovascular, San Jose, Calif; and MicroPlex, MicroVention, Aliso Viejo, Calif; Fig $1 B$ ). The packing attenuation following coiling was calculated at $13 \%$. During the ensuing 24 hours, the patient developed visual scintillations in the right eye, which were consistent with his well-documented migraine auras. A follow-up angiogram 1 day following the coil procedure showed complete aneurysm occlusion and no evidence for parent artery compromise or distal emboli. On the third hospital day, the patient was dismissed home and was maintained on aspirin and clopidogrel.

Approximately 1 week after the coiling, the patient presented to his local hospital with symptoms of headache, dysequilibrium, abducens palsy, and internuclear ophthalmoplegia. An MR imaging showed the coiled aneurysm in the setting of marked brain stem edema (Fig 1C). At that time, angiographic findings were unchanged as compared with the patient's angiogram obtained at the time of his coiling. Corticosteroids were initiated, but during the course of the following 5 days, the patient developed hydrocephalus, necessitating placement of an external ventricular drain, as well as a posterior fossa hemorrhage. His neurologic status continued to decline with the family withdrawing support on postcoiling day 18 .

Received September 25, 2007; accepted after revision October 11.

From the Departments of Neurological Surgery (J.B.W.) and Radiology (H.J.C., D.F.K.), Mayo Clinic, Rochester, Minn.

Please address correspondence to David F Kallmes, MD, Mayo Clinic, Department of Radiology, 200 First St SW, Rochester, MN 55905; e-mail: kallmes.david@mayo.edu

DOI 10.3174/ajnr.A0877

\section{Case 2}

The patient was a 55-year-old man who presented clinically as a Hunt and Hess grade 1. CT imaging revealed a Fisher grade 3 subarachnoid hemorrhage with no evidence for cerebral or brain stem edema. Angiography revealed an aneurysm at the basilar apex measuring $13 \times$ $10 \times 9 \mathrm{~mm}$ (Fig $2 \mathrm{~A}$ ). Under general anesthesia, he underwent successful coiling of the aneurysm with a total of 12 bare platinum coils (MicroPlex, MicroVention; Fig 2B). The calculated packing attenuation of the aneurysm following coiling was $24 \%$.

On the second day of his hospitalization, an external ventricular drain was placed for hydrocephalus, which was subsequently replaced with a ventriculoperitoneal shunt. For 1 month following the patient's coiling procedure, he experienced significant hypersomnolence, which fluctuated with near-complete resolution before discharge. An MR imaging was performed 6 days following the coil procedure, which was remarkable only for profound edema within the brain stem adjacent to the coiled aneurysm (Fig 2C). Vasospasm surveillance by using transcranial Doppler sonography and angiography revealed no evidence for altered flow velocities. At 5 weeks from his initial hemorrhage, the patient was discharged to an inpatient rehabilitation unit, where he thrived until discharge to his home.

\section{Discussion}

This report shows that patients with aneurysms coiled with bare platinum alone are capable of developing the same clinical sequelae that have been previously identified with the use of bioactive coils. ${ }^{1-4}$ Both of our patients developed significant perianeurysmal edema, which was manifest in their brain stems. Both of our patients also developed hydrocephalus, one of whom did so in the setting of an unruptured aneurysm. Both patients developed a fluctuating level of consciousness that ultimately resolved in one and led to hemorrhage and death in another.

In previous cases in which patients were coiled with bioactive materials, the symptoms and signs at presentation were commonly headache, malaise, and cranial neuropathies. ${ }^{1-4}$ Other findings were fever, nuchal rigidity, visual loss, and photophobia. In nearly every patient, corticosteroids were implemented with near-complete resolution of the patients' symptoms. Despite the use of steroids, the condition of one of our patients (case 1) continued to decline, with a subsequent hemorrhage and ultimately death. The etiology of hemorrhage in this patient is unclear, though one may conclude that the aneurysm ruptured in the setting of vast inflammation coupled with fluctuating transmural pressures associated with the development and treatment of his hydrocephalus. In the other patient (case 2), steroids were not implemented; rather, stimulants were used, leading to a gradual improvement. 

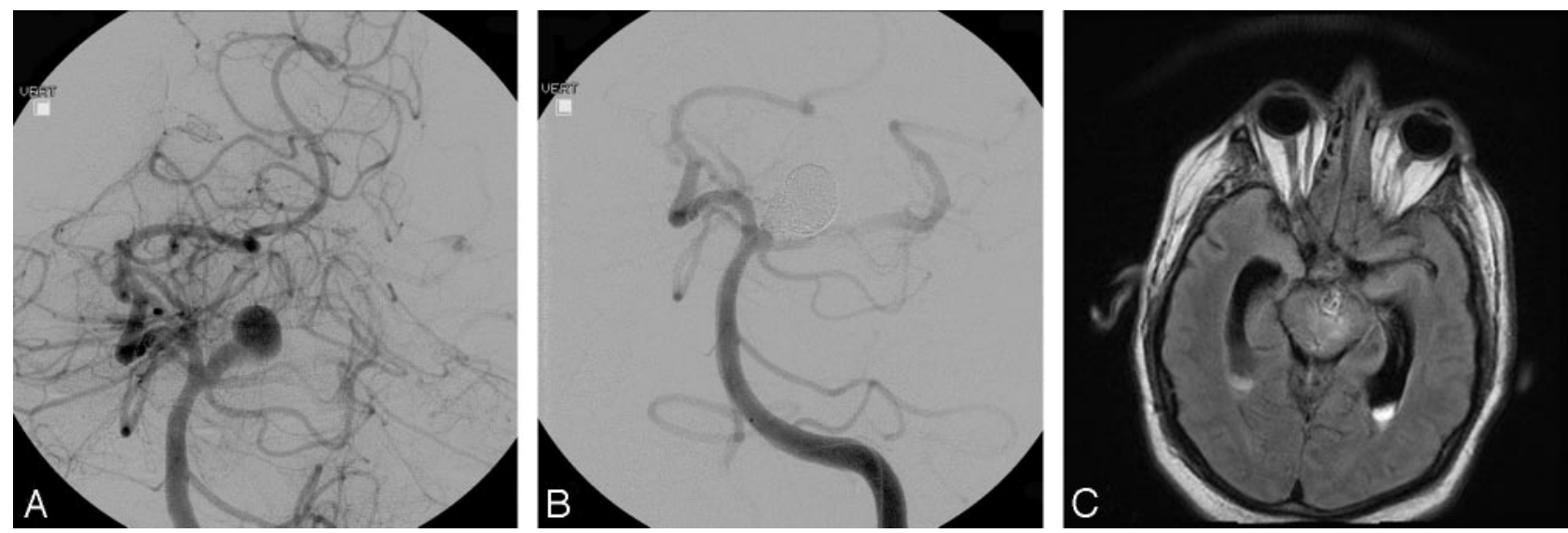

Fig 1. $A$, Pre-embolization angiogram showing a large right vertebrobasilar region aneurysm. $B$, Postembolization angiogram showing the coiled aneurysm with minimal residual neck. $C$ Axial flare MR image showing edema within the brain stem adjacent to the coiled aneurysm.
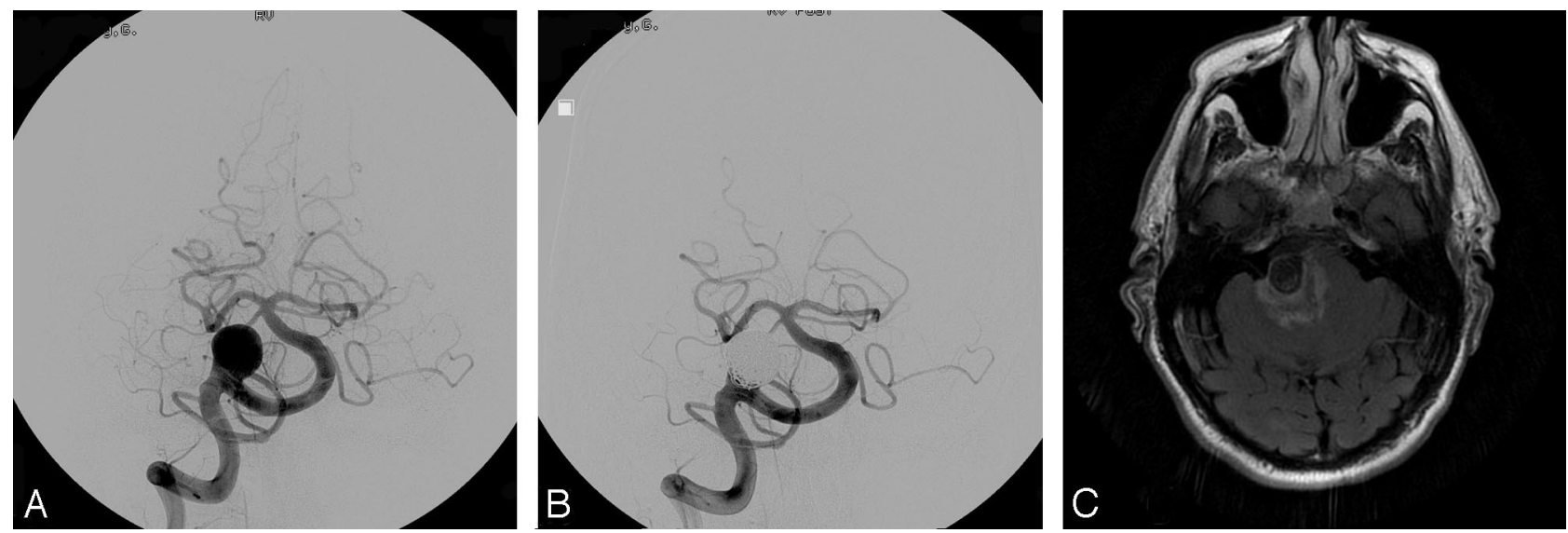

Fig 2. A, Pre-embolization angiogram showing a large basilar apex region aneurysm. $B$, Postembolization angiogram showing the coiled aneurysm with minimal residual neck. A large posterior communicating artery on the left produces washout of the left posterior cerebral artery. $C$, Axial flare MR image showing edema within the brain stem adjacent to the coiled aneurysm.

The development of hydrocephalus has also been well documented in cases of unruptured aneurysms following treatment with bioactive coils. ${ }^{1-4}$ Some have proposed that the development of an aseptic meningitis and its inflammatory sequelae leads to a communicating form of hydrocephalus. ${ }^{3}$ This would seem a plausible explanation for the hydrocephalus that developed in our first patient. The development of hydrocephalus in the same set of treated patients with ruptured aneurysms is more difficult to interpret because a substantial percentage of those patients will also develop hydrocephalus in the absence of endovascular therapy. In the recently completed HELPS (HydroCoil Endovascular Aneurysm Occlusion and Packing Study), early hydrocephalus ( $<3$ months post coiling) occurred in a small number of randomized unruptured aneurysms in both arms of the trial with no significant difference identified between the HydroCoil and control arms ( $p=0.6$ ) (unpublished data by P. White presented on behalf of HELPS trialists, World Federation of Interventional and Therapeutic Neuroradiology meeting, September 2007, Beijing, China).

A review of the recent reports highlighting the complications of hydrocephalus, aseptic meningitis, and perianeurysmal edema from coiling 11 aneurysms reveals that a substantial number of the aneurysms treated were large $(6,8,8,8,10$, $11,15,15,16,20$, and $20 \mathrm{~mm}) \cdot{ }^{1-5}$ It may be that the natural inflammatory response that occurs with the healing of aneurysms is proportional to the size of the aneurysm treatedboth of the patients in our report harbored large aneurysms.

We conclude that adverse inflammatory reactions from coiling of aneurysms are not limited to bioactive coils. Bare platinum coils can also lead to perianeurysmal edema and hydrocephalus.

\section{Acknowledgment}

The authors acknowledge Nils Mueller-Kronast, MD, Director of Neurointerventional Radiology, Parkview Health, Ft. Wayne, Ind for providing images for this case report.

\section{References}

1. Deshaies EM, Adamo MA, Boulos AS. A prospective single-center analysis of the safety and efficacy of the HydroCoil embolization system for the treatment of intracranial aneurysms. J Neurosurg 2007;106:226-33

2. Im SH, Han $\mathrm{MH}$, Kwon BJ, et al. Aseptic meningitis after embolization of cerebral aneurysms using hydrogel-coated coils: report of three cases. AJNR Am J Neuroradiol 2007;28:511-12

3. Stracke CP, Krings T, Moller-Hartmann W, et al. Severe inflammatory reaction of the optic system after endovascular treatment of a supraophthalmic aneurysm with bioactive coils. AJNR Am J Neuroradiol 2007;28:1401-02

4. Meyers PM, Lavine SD, Fitzsimmons BF, et al. Chemical meningitis after cerebral aneurysm treatment using two second-generation aneurysm coils: report of two cases. Neurosurgery 2004;55:1222

5. Horie N, Kitagawa N, Morikawa M, et al. Progressive perianeurysmal edema induced after endovascular coil embolization: report of three cases and review of the literature. J Neurosurg 2007;106:916-20 